\title{
A Classification of Open String Models
}

\author{
W. Nahm
}

Physikalisches Institut der Universität Bonn, Nussallee 12, D-5300 Bonn 1, Federal Republic of Germany

\begin{abstract}
Open string models are classified using modular invariance. No good candidates for new models are found, though the existence of an $E_{8}$ invariant model in $R^{17,1}$, a similar one in $R^{5,1}$ and of a supersymmetric model in $R^{2,1}$ cannot be excluded by this technique. An intriguing relation between the left moving and right moving sectors of the heterotic string emerges.
\end{abstract}

Due to the conformal invariance of string theories, quantities defined by integrals over function spaces defined on the string world sheet can be described by holomorphic forms in Teichmüller space, i.e. the space of classes of conformally equivalent metrics on a compact Riemann surface of given genus. For genus $g=1,2,3$ this space is given by the coset space $H(g) / \mathrm{Sp}(g, \mathbb{Z})$, where $H(g)$ is the Siegel upper half plane of dimension $g(g+1) / 2$. Thus one obtains holomorphic forms on $H(g)$ which are invariant under $\operatorname{Sp}(g, \mathbb{Z})$.

In particular this applies to various partition functions using light cone variables which can be defined as suitable traces or supertraces in the Hilbert space of a non-interacting string $[1,2]$. Such a trace is given by a functional integral with the condition that the states of the string at light cone times $t_{0}$ and $t_{1}$ are the same. For closed strings a state at time $t$ is specified by functions $f(\sigma, t), \sigma \in R \bmod 2 \pi$, but for constant $\sigma_{0}$ the functions $f(\sigma, t)$ and $f\left(\sigma+\sigma_{0}, t\right)$ denote the same state. This introduces a complication which will not be discussed in the present paper, which deals exclusively with open strings.

Open strings are described by functions $f(\sigma, t), \sigma \in[0, \pi]$. For functional integrals yielding traces one must have $f\left(\sigma, t_{0}\right)=f\left(\sigma, t_{1}\right)$, as the boundaries at $\sigma=0, \pi$ are fixed. The string surface over which one integrates then is the annulus $[0, \pi] \times\left(R \bmod \left(t_{1}-t_{0}\right)\right)$. However, one can go over to a compact double cover of this annulus, namely the torus $(R \bmod 2 \pi) \times\left(R \bmod \left(t_{1}-t_{0}\right)\right)$, with projection $\sigma \rightarrow|\sigma|$. Physically, the double covering corresponds to the separation of right moving and left moving excitations of the string, which due to the conformal invariance do not interact at all, apart from the boundaries, where they are reflected and transform into each other. 
For a functional integral over the annulus, the normal modes $\exp (i n \sigma)$ and $\exp (-i n \sigma)$ are related by an involution. In the corresponding unrestricted functional integral over the torus, this restriction is removed. As the contributions of different normal modes are independent, the torus integral is just the square of the annulus integral, apart from the zero mode factor with $n=0$, which is not squared. Up to this zero mode factor open string partition functions are given by the square root of integrals over functions on a torus.

Tori have genus $g=1$, and the corresponding $H(1)$ just is the ordinary complex upper half plane with coordinate $w$, which continues $i\left(t_{1}-t_{0}\right) / 2 \pi$ to complex values with arbitrary real part. On $H(1)$ the modular group $\operatorname{Sp}(1, \mathbb{Z}) / \mathbb{Z}_{2}$ $=\mathrm{SL}(2, \mathbb{Z}) / \mathbb{Z}_{2}$ acts by

$$
w \rightarrow \frac{a w+b}{c w+d}, \quad\left(\begin{array}{ll}
a & b \\
c & d
\end{array}\right) \in \operatorname{SL}(2, \mathbb{Z}) .
$$

The invariance under the modular group can be used to classify string models by specifying the partition functions for the non-interacting string. This does not necessarily yield a unique determination of the model, as e.g. the two possible bosonic sectors of the heterotic string [3] with $E_{8} \times E_{8}$ and $\operatorname{Spin}^{+}$(32) symmetry respectively have identical partition functions, such that the existence of two different models was not recognized, when this partition function first turned up in a string model classification [2]. Moreover, the method yields no constraints on Chan-Paton variables attached to the ends of the string, as these do not change the dynamics of the non-interacting string. Nevertheless, the following results indicate that for open strings new models may not exist within the presently established framework.

Consider an open, non-interacting string in $R^{D+1,1}$. For fixed light cone momentum $p_{+}$and zero transverse momentum, all states of the string can be classified according to their mass $m$ and their transformation properties under the transverse rotation group $\mathrm{SO}(D)$ or rather its double covering $\operatorname{Spin}(D)$. Let $r$ be the rank of $\operatorname{Spin}(D)$ and $T(\operatorname{Spin}(D))=R^{r} / \Lambda$ its maximal torus, such that $\Lambda$ is the weight lattice of $\operatorname{Spin}(D)$. For each weight $\lambda \in \Lambda$, let $d_{B}(m, \lambda)$ be the number of bosonic states with mass $m$ and helicity $\lambda$ and $d_{F}(m, \lambda)$ the corresponding number of fermionic states. Of course $d_{B}(m, \lambda)$ only can be positive for weights of $\operatorname{SO}(D)$ and $d_{F}(m, \lambda)$ only for the remaining weights of $\operatorname{Spin}(D)$.

If no fermions are present, the annulus functional integral yields an ordinary trace, but with fermions one obtains a supertrace. Thus we define

$$
d(m, \lambda)=d_{B}(m, \lambda)-d_{F}(m, \lambda), \quad d(m)=\sum_{\lambda \in \Lambda} d(m, \lambda),
$$

and the partition function

$$
A(w)=\sum_{m} d(m) \exp \left(2 \pi i w m^{2}\right),
$$

where masses are measured in some suitable unit. For physically reasonable mass spectra $A(w)$ is well defined and regular in the interior of $H(1)$. 
Let $\zeta=\left(\zeta_{1}, \ldots, \zeta_{r}\right)$ parametrize the maximal torus $R^{r} / \mathbb{Z}^{r}=\mathrm{SO}(2)^{r}$ of $\mathrm{SO}(D)$ and its double covering $T(\operatorname{Spin}(D))$. Define the helicity partition function

$$
A(\zeta, w)=Z(\zeta) \sum_{m} \sum_{\lambda \in A} d(m, \lambda) \exp \left(2 \pi i \zeta \lambda+2 \pi i w m^{2}\right) .
$$

Here we use zero mode factor [2]

$$
Z(\zeta)=\operatorname{det}\left(\frac{d}{d t}+2 \pi \zeta_{i} L^{i} /\left(t_{1}-t_{0}\right)\right)^{-1}=\prod_{i=1}^{r}\left(2 \sin \pi \zeta_{i}\right)^{-1},
$$

where $L^{i}, i=1, \ldots, r$ denotes the Lie derivatives given by the generators of $T(\operatorname{Spin}(D))$ and the differential operator acts on the maps from $\left(R \bmod \left(t_{1}-t_{0}\right)\right)$ to $R^{D}$. Note that $Z(\zeta)$ is double valued on $\mathrm{SO}(2)^{r}$, but single valued on $T(\operatorname{Spin}(D))$.

For physically reasonable $d(m, \lambda)$, analytic continuation in $\zeta$ yields a meromorphic function $A(\zeta, w)$ defined on $C^{r} / \Lambda \times H(1)$. The relevant transformation law for helicity partition functions is that of Jacobi forms $[2,4]$.

Definition. A meromorphic function $F(\zeta, w)$ on $C^{r} \times H(1)$ is a Jacobi form of weight $\alpha \in \mathbb{Z} / 2$ and index $k \in \mathbb{C}$ with respect to a lattice $\Lambda \subset R^{r}$, if

1) as function of $q^{1 / 2}=\exp (\pi i w)$ it is meromorphic in a neighbourhood of $q^{1 / 2}=0$,

2) $F(\zeta+\lambda, w)=F(\zeta, w)$ for $\lambda \in \Lambda$,

3) for arbitrary $\left(\begin{array}{ll}a & b \\ c & d\end{array}\right) \in \operatorname{SL}(2, \mathbb{Z})$ it satisfies

$$
F\left(\frac{\zeta}{c w+d}, \frac{a w+b}{c w+d}\right)=\varepsilon(c w+d)^{\alpha} F(\zeta, w) \exp \left(k \pi i \frac{c \zeta^{2}}{c w+d}\right)
$$

with $\varepsilon \in\{+1,-1\}$.

In the degenerate case $r=0$ we call $F(w)$ a modular form, as usual.

In the terminology of [4] our $F(\zeta, w)$ might more precisely be called meromorphic Jacobi forms.

We shall not distinguish between functions defined on $C^{r} / \Lambda$ and functions defined on $C^{r}$ with period lattice $\Lambda$. Thus the helicity partition functions are candidates for Jacobi forms. We tentatively admit sign factors $\varepsilon$, as our partition functions are given by square roots of functional integrals on tori. The functional form of the cocycle in the Jacobi form transformation law is the only possible one [5]. A cocycle appears, when the relevant functional integrals are sections of nontrivial line bundles over $C^{r} /(\Lambda+w \Lambda)$. This happens e.g. for [6]

$$
\operatorname{det}\left(\frac{\partial^{2}}{\partial \sigma^{2}}+\left(\frac{\partial}{\partial t}+2 \pi \zeta_{i} L^{i} /\left(t_{1}-t_{0}\right)\right)^{2}\right) .
$$

If one expands the logarithm of this determinant in powers of $\zeta$, the coefficient of $\zeta^{2}$ is logarithmically divergent, which explains the form of the cocycle. This phenomenon is general, because the operator multiplying $\zeta$ must be of dimension 1 to yield an $\mathrm{SO}(D)$ symmetry current, whereas the $\zeta$-independent operator yields an energy-momentum density and must have dimension 2 . It is easy to see that the cocycle index $k$ is real and quantized. 
Lemma 1. For a Jacobi form of index $k$ with respect to the lattice $\lambda$

$$
F\left(\zeta+\lambda w+\lambda^{\prime}, w\right)=F(\zeta, w) \exp \left(-k \pi i\left(2 \zeta \lambda+w \lambda^{2}\right)\right),
$$

and

$$
k \lambda^{2} \in \mathbb{Z}
$$

for all $\lambda, \lambda^{\prime} \in \Lambda$.

Proof. Conjugation of a $\Lambda$ translation by the modular transformation $w \rightarrow(-1 / w)$ yields the first formula. Applying the transformation $w \rightarrow(w+1)$ to this equation yields the second one.

If $A(\zeta, w)$ is a Jacobi form of weight $\alpha$, then $A(w)$ is a modular form of weight $\alpha-r$. This weight can be determined as follows [7]. On strings no long range correlations should exist, such that for fixed $L^{\prime} \in(0, L)$ the space of strings of length $L$ modulo translation should essentially be the tensor product of the corresponding spaces for lengths $L$ and $L-L^{\prime}$. Certain quantum numbers like momentum are distributed additively among the two parts. A natural measure for the length of the string is the light cone momentum $p_{+}=\left(p_{0}-p_{1}\right) / 2$. Let $\varrho(p)$ be the density of string states with momentum $p$. Then one expects

$$
\varrho\left(p_{+}, p_{-}, p_{\perp}\right) \simeq \int d p_{-}^{\prime} d p_{\perp}^{\prime} \varrho\left(p_{+}-p_{+}^{\prime}, p_{-}-p_{-}^{\prime}, p_{\perp}-p_{\perp}^{\prime}\right) \varrho\left(p_{+}^{\prime}, p_{-}^{\prime}, p_{\perp}^{\prime}\right) .
$$

For the Laplace transform in $p_{-}=\left(p_{\perp}^{2}+m^{2}\right) / 2 p_{+}$,

$$
\int \varrho\left(p_{+}, p_{-}, p_{\perp}\right) \exp \left(-p_{-} t\right) d p_{-} d p_{\perp}=\left(2 \pi p_{+} / t\right)^{D / 2} A\left(i t / 2 \pi p_{+}\right),
$$

one obtains

$$
\left(2 \pi p_{+} / t\right)^{D / 2} A\left(i t / 2 \pi p_{+}\right) \simeq \exp \left(c p_{+} / t\right)
$$

for some constant $c$, which yields a weight factor $w^{-D / 2}$ for the transformation from $A(w)$ to $A(-1 / w)$. Thus the weight of $A(\zeta, w)$ should be $r-D / 2$, i.e. zero for even $D$ and $-1 / 2$ for odd $D$.

Actually $w^{-D / 2} A(w)$ should be a sum of exponentials in $-1 / w$ with integral coefficients. Our argument just has recovered the dominant term, but along the lines of [7] it might be possible to explain the whole series.

Another physical argument can be used to control the poles of $A(\zeta, w)$. As the zero mode factor $Z(\zeta)$ has single poles at $\zeta \in \mathbb{Z}^{r}$, Lemma 1 yields single poles at all points of the lattice $\mathbb{Z}^{r}+w \mathbb{Z}^{r}$. Poles in $\zeta$ correspond to the linear Regge trajectories of the string [2]. The leading trajectory is given by rigid rotations in $R^{D}$, as for a string with length $L$ and total momentum $p=(E, 0)$ one has a geometric angular momentum $J \lesssim L E \lesssim E^{2}=m^{2}$, whereas internal contributions to the angular momentum should only be proportional to $E$. Thus changes in the internal structure of a string only should affect the Regge intercepts. In particular the poles of $A(\zeta, w)$ should be independent of the details of the string structure, and no new poles should occur besides the ones at $\mathbb{Z}^{r}+w \mathbb{Z}^{r}$, which are due to the basic kinematics. When we divide $A(\zeta, w)$ by the standard bosonic string contribution, the resulting reduced helicity partition function,

$$
H(\zeta, w)=\eta^{-3 r}(w) \theta_{1}^{r}(\zeta, w) A(\zeta, w),
$$

thus should be holomorphic in $C^{r} \times H(1)$. 
We use the standard notations

$$
\begin{gathered}
q^{1 / 24}=\exp (\pi i w / 12), \quad \eta(w)=q^{1 / 24} \prod_{n=1}^{\infty}\left(1-q^{n}\right) \\
\theta_{1}(z, w)=2 q^{1 / 12} \eta(w) \sin \pi z \prod_{n=1}^{\infty}\left(1-e^{2 \pi i z} q^{n}\right)\left(1-e^{-2 \pi i z} q^{n}\right) \\
\theta_{2}(z, w)=2 q^{1 / 12} \eta(w) \cos \pi z \prod_{n=1}^{\infty}\left(\left(1+e^{2 \pi i z} q^{n}\right)\left(1+e^{-2 \pi i z} q^{n}\right)\right) \\
\theta_{3}(z, w)=q^{-1 / 24} \prod_{n=1}^{\infty}\left(\left(1 \pm e^{2 \pi i z} q^{n-1 / 2}\right)\left(1 \pm e^{-2 \pi i z} q^{n-1 / 2}\right)\right) \eta(w)
\end{gathered}
$$

and

$$
\theta_{j}^{r}(\zeta, w)=\prod_{i=1}^{r} \theta_{j}\left(\zeta_{i}, w\right), \quad j=1,2,3,4 .
$$

Note that $\eta^{-3 r}(w) \theta_{1}^{r}(\zeta, w)$ is a Jacobi form of weight $-r$ and index 1 with respect to $\Lambda$, such that $H(\zeta, w)$ is a Jacobi form of weight $-D / 2$.

The dominant Regge intercepts are determined by the index $k$ of $H(\zeta, w)$. For $k>1$ one obtains sister Regge trajectories of arbitrarily high intercept [2], which for the corresponding interacting string theory should be incompatible with unitarity. The snag with this argument is that closed string theories apparently reconcile unitarity at least with a graviton trajectory of intercept 2 . Thus the existence of a loophole for $k>1$ is perhaps conceivable, but this possibility will be ignored in the following classification.

Theorem. Let $H(\zeta, w)$ be reduced helicity partition function of a Lorentz invariant string theory in $R^{D+1,1}$ with at most a finite number of tachyon states. Let $H(\zeta, w)$ be holomorphic in $C^{r} \times H(1)$ and transform as a Jacobi form of weight $-D / 2$ and index $k \leqq 1$ with respect to the weight lattice $\Lambda$ of $\operatorname{Spin}(D)$. Then $H(\zeta, w)$ can be obtained by integral linear combination from the following functions:

For $\varepsilon \equiv 1$ :

$$
\begin{array}{ll}
D=24, k=0: & H_{26}(\zeta, w)=\eta^{-24}(w), \\
D=16, k=0: & H_{18}(\zeta, w)=\eta^{-16}(w) \chi(w), \\
D=8, k=0: & H_{10}^{B}(\zeta, w)=\eta^{-8}(w) \chi(w)^{2}, \\
D=8, k=1: & H_{10}^{ \pm}(\zeta, w)=\frac{1}{2} \eta^{-12}(w)\left[ \pm \theta_{1}^{4}(\zeta, w)-\theta_{2}^{4}(\zeta, w)+\theta_{3}^{4}(\zeta, w)-\theta_{4}^{4}(\zeta, w)\right], \\
D=0: & H_{2}^{n}(w)=\chi(w)^{n}, \quad n=0,1,2, \ldots,
\end{array}
$$

for $\varepsilon \neq 1$ :

$$
\begin{array}{ll}
D=4, k=0: & H_{6}(\zeta, w)=\eta^{-4}(w) \chi(w), \\
D=0: & H_{2}^{\prime n}(w)=-\eta^{-12}(w) E_{6}(w) \chi(w)^{n}, \quad n=0,1,2, \ldots,
\end{array}
$$

and finally

Here

$$
D=1: H_{3}(w)=0 \text {. }
$$

$$
E_{6}(w)=1-504 \sum_{n=1}^{\infty} \frac{n^{5} q^{n}}{1-q^{n}},
$$


and

$$
\chi(w)=\eta^{-8}(w) E_{4}(w)=\eta^{-8}(w)\left(1+240 \sum_{n=1}^{\infty} \frac{n^{3} q^{n}}{1-q^{n}}\right) .
$$

The lower index of $H$ in this list denotes the space-time dimension $D+2$. For $D=26$ the list yields the standard bosonic string. For $D=8,16$ note that $\chi(w)$ is the character of the basic representation of the euclidean Kac-Moody algebra $E_{8}^{(1)}$ [8]. Its square can be interpreted as character of $E_{8}^{(1)} \times E_{8}^{(1)}$ or $D_{16}^{(1)}$. Thus $H_{10}^{B}$ is the partition function of the bosonic sector of the heterotic string. $H_{10}^{ \pm}$is the reduced helicity partition of the superstring for chirality \pm . Its notation in the theorem corresponds to its original construction from the Neveu-Schwarz and Ramond strings [9]. With the triality transformations,

$$
\xi^{ \pm}=\frac{1}{2}\left(\zeta_{1}+\zeta_{2}+\zeta_{3} \pm \zeta_{4}, \zeta_{1}+\zeta_{2}-\zeta_{3} \mp \zeta_{4}, \zeta_{1}-\zeta_{2}+\zeta_{3} \mp \zeta_{4}, \zeta_{1}-\zeta_{2}-\zeta_{3} \pm \zeta_{4}\right),
$$

one may write

$$
H_{10}^{ \pm}(\zeta, w)=\eta^{-12}(w) \theta_{1}^{4}\left(\xi^{ \pm}, w\right),
$$

which corresponds to the formalism of Green and Schwarz [10].

For $D>1$ the function $H_{6}$ is the only one in the list which transforms with a non-trivial phase factor $\varepsilon$. Though $\chi(w)$ is the basic $E_{8}^{(1)}$ character, $H_{6}$ cannot come from a theory with unbroken $E_{8}$ symmetry, as otherwise the $\mathrm{SO}(4)$ vector multiplet at $m^{2}=1 / 2$ would lack a singlet to complete the $\mathrm{SO}(5)$ vector which is required by Lorentz invariance.

One has to list the possibility $H_{3}(w)=0$, as for $D \leqq 1$ bosonic and fermionic contributions to the partition function cannot be distinguished, such that a supersymmetric string model in $R^{2,1}$ cannot be ruled out by the present approach.

For $D=0$ one just expects partition functions of conformally invariant field theories in $1+1$ dimensions, with space compactified by suitable boundary conditions. A free scalar field taking values on an $8 n$ dimensional torus with even integral, self-dual period lattice yields, e.g. a linear combination of the $H_{2}^{m}$, $m=0, \ldots, n$.

The proof of the theorem will be given as a series of lemmata.

Lemma 2. If $D \notin 4 \mathbb{Z}, H(\zeta, w)$ vanishes.

Proof. For $w$ purely imaginary and $\zeta$ either real or purely imaginary, $H(\zeta, w)$ is real. Comparing the phases after a modular transformation $w \rightarrow(-1 / w)$, one finds that $i^{D / 2}$ must be real.

Lemma 3. $k \in\{0,1\}$.

Proof. For $D \geqq 4$ the weight lattice of $\mathrm{SO}(D)$ contains weights $\lambda$ with $\lambda^{2}=1$. Thus Lemma 1 yields $k \in \mathbb{Z}$. Applying Cauchy's theorem to $H^{-1} d H / d \zeta_{i}$, one finds that $k$ is non-negative. The restriction $k \leqq 1$ has been stated in the assumption.

Lemma 4. For the sign factor $\varepsilon$ in the transformation law

$$
H\left(\frac{\zeta}{c w+d}, \frac{a w+b}{c w+d}\right)=\varepsilon\left(\begin{array}{ll}
a & b \\
c & d
\end{array}\right)(c w+d)^{-D / 2} H(\zeta, w) \exp \left(k \pi i \frac{c \zeta^{2}}{c w+d}\right)
$$


one has the two possibilities

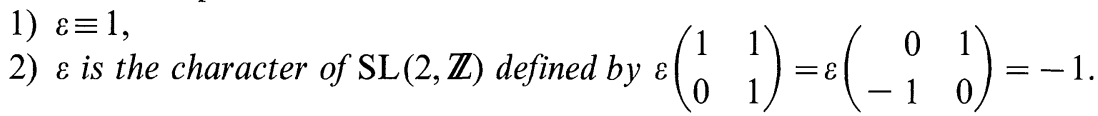

Proof. $\varepsilon$ must be a character of $\operatorname{SL}(2, \mathbb{Z})$ which is trivial on all group elements generated by squares, in particular on

$$
\operatorname{SL}(2, \mathbb{Z}, 2)=\left\{\left(\begin{array}{ll}
a & b \\
c & d
\end{array}\right) \in \operatorname{SL}(2, \mathbb{Z}) \mid\left(\begin{array}{ll}
a & b \\
c & d
\end{array}\right)=\left(\begin{array}{ll}
1 & 0 \\
0 & 1
\end{array}\right) \bmod 2\right\} .
$$

The quotient group $\operatorname{SL}(2, \mathbb{Z}) / \operatorname{SL}(2, \mathbb{Z}, 2)$ is the symmetric group $S_{3}$, which just has the two homomorphisms into $\mathbb{Z}_{2}$ used above.

Lemma 5. $H(\zeta, w)$ can be extended to the compactification of $H(1) / \operatorname{SL}(2, \mathbb{Z})$ given by the local coordinate $q$ in the neighbourhood of $w=i \infty$. For $\varepsilon \equiv 1, H(\zeta, w)$ is meromorphic in $q$, for $\varepsilon$ 丰 1 the same is true for $q^{1 / 2} H(\zeta, w)$.

Proof. This is a direct consequence of the assumption that there are at most a finite number of tachyonic states and of the behaviour under $w \rightarrow w+1$.

Lemma 6. For $k=0$ or $D=0$ one obtains the list of possibilities given in the theorem.

Proof. For $k=0$ the reduced helicity partition function is a doubly periodic holomorphic function in all components of $\zeta$, i.e. $H(\zeta, w)=A(w)$. In particular, the string is purely bosonic. Define $N$ by $A(w) \sim q^{-N}$ at $q=0$, where $N \in \mathbb{Z}$ for $\varepsilon \equiv 1$ and $N \in \mathbb{Z}+1 / 2$ for $\varepsilon \neq 1$. Let $n_{0}$ be the number of zeros of $A(w)$ at generic positions in $H(1) / \mathrm{SL}(2, \mathbb{Z})$ and $n_{2}, n_{3}$ the degrees of zeros at $\exp (\pi i / 2), \exp (\pi i / 3)$. Then [11]

$$
N-n_{0}-n_{2} / 2-n_{3} / 3=D / 24 \text {. }
$$

Because of Lorentz invariance, tachyons form unitary representations of $\operatorname{Spin}(D, 1)$. These are infinite dimensional or trivial. Thus $d(m, \lambda)=0$ for $m^{2}<0$, $\lambda \neq 0$. For $D>0$ this yields $N \leqq 1$, and consequently $n_{0}=0$. Thus $A(w)$ is given by a suitable product of powers of the holomorphic modular forms $\eta^{24}(w), E_{4}(w)$, $E_{6}(w)$, which have simple zeros at $i \infty, \exp (\pi i / 2), \exp (\pi i / 3)$ respectively and nowhere else. The cases with $n_{2}=0$ have been listed in the theorem, with the exception of

$$
H_{14}(\zeta, w)=\eta^{-12}(w),
$$

which violates Lorentz invariance, as it does not yield a representation of $\mathrm{SO}(13)$ at $m^{2}=1 / 2$. Nevertheless the corresponding bosonic string in 14 dimensions is of some interest, as compactification of 4 dimensions on $\mathbb{Z}^{4}$ leads to the superstring [12].

A zero at $w=i$ is incompatible with $d(m, \lambda)=d_{B}(m, \lambda) \geqq 0$. In fact for $n_{2}=1$ one obtains

$$
\begin{array}{ll}
D=12: & H_{14}^{B g}(\zeta, w)=-\eta^{-24}(w) E_{6}(w), \\
D=4: & H_{6}^{B g}(\zeta, w)=-\eta^{-24}(w) E_{4}(w) E_{6}(w) .
\end{array}
$$

In these cases, $d(m, 0)=-1$ for $m^{2}=-1$. Perhaps there are corresponding models with tachyonic ghosts, which might have some use. The $B$ in $H^{B g}$ stands for bosonic, the $g$ for ghost. 
For $D=0$, one considers the holomorphic modular form $\eta^{24 N}(w) A(w)$, which is of weight $12 N$ and has $\varepsilon \equiv 1$. All such forms are polynomials in $E_{4}(w), E_{6}(w)$ [11]. As $E_{4}(w)^{3}-E_{6}(w)^{2}$ is proportional to $\eta^{24}(w)$, one obtains the list given in the theorem.

Lemma 7. For $k=1$ one has

$$
H(\zeta, w)=\eta^{3 r}(w) \sum_{j=1}^{4} h_{j}(w) \theta_{j}^{r}(\zeta, w) .
$$

Here $h_{1}(w), h_{j}(w)$ are modular of weight 0 for $\operatorname{SL}(2, \mathbb{Z}), \operatorname{SL}(2, \mathbb{Z})_{j}, j=2,3,4$, where

$$
\begin{aligned}
& \operatorname{SL}(2, \mathbb{Z})_{2}=\left\{\left(\begin{array}{ll}
a & b \\
c & d
\end{array}\right) \in \operatorname{SL}(2, \mathbb{Z}) \mid c \equiv 0 \bmod 2\right\} \\
& \mathrm{SL}(2, \mathbb{Z})_{3}=\left\{\left(\begin{array}{ll}
a & b \\
c & d
\end{array}\right) \in \operatorname{SL}(2, \mathbb{Z}) \mid a+b+c+d \equiv 0 \bmod 2\right\} \\
& \mathrm{SL}(2, \mathbb{Z})_{4}=\left\{\left(\begin{array}{ll}
a & b \\
c & d
\end{array}\right) \in \mathrm{SL}(2, \mathbb{Z}) \mid b \equiv 0 \bmod 2\right\}
\end{aligned}
$$

are conjugate subgroups of $\mathrm{SL}(2, \mathbb{Z})$. The $h_{j}(w)$ are related by the same conjugations.

Proof. With respect to each $\zeta_{i}, H(\zeta, w)$ is a holomorphic section of a line bundle over $C / 2(\mathbb{Z}+w \mathbb{Z})$, with cocycle given by Lemma 1 . For $k=1$ these sections are linear combinations of the $\theta_{j}\left(\zeta_{i}, w\right), j=1,2,3,4$. Imposing periodicity of $H(\zeta, w)$ on $\Lambda$ one finds that $H(\zeta, w)$ must be a linear combination of the $\theta_{j}^{r}(\zeta, w)$. Applying modular transformations of all cosets $\operatorname{SL}(2, \mathbb{Z}) / \operatorname{SL}(2, \mathbb{Z}, 2)$ one obtains the remaining statements of the lemma

Lemma 8. For $k=1, H(\zeta, w)$ must be the reduced partition function of a superstring.

Proof. Let $H_{j}(w) \sim q^{-N j}$ at $q=0$. Invariance under $w \rightarrow w+1$ yields $N_{3}=N_{4} \in \mathbb{Z} / 2+D / 16 ; N_{1}, N_{2} \in \mathbb{Z}$ for $\varepsilon \equiv 1 ; N_{1}, N_{2} \in \mathbb{Z}+1 / 2$ for $\varepsilon \neq 1$. To avoid tachyon states transforming non-trivially under $\operatorname{Spin}(D)$ one must have $N_{1} \leqq 0$, $N_{2} \leqq 0, N_{4} \leqq 1-D / 16$ for $\varepsilon \equiv 1$ and $N_{1} \leqq-1 / 2, N_{2} \leqq-1 / 2, N_{4} \leqq 1 / 2-D / 16$ for $\varepsilon \neq 1$.

In particular, $h_{1}(w)$ is a modular form of weight 0 without poles, i.e. a constant, which even must vanish for $\varepsilon$ 三 1 .

In the fundamental domain $H(1) / \mathrm{SL}(2, \mathbb{Z})_{2}$ of $h_{2}(w)$ there are two inequivalent cusps at $w=0, i \infty$, and the special point $w=(1+i) / 2$, which is left invariant under the involution $\left(\begin{array}{ll}1 & -1 \\ 2 & -1\end{array}\right)$ of $\operatorname{SL}(2, \mathbb{Z})_{2} / \mathbb{Z}_{2}$. Using the transformation $w \rightarrow(-1 / w)$ one sees that $h_{2}(w) \sim \hat{q}^{-N_{4}}$ at $\hat{q}=0$, where $\hat{q}=\exp (-2 \pi i / w)$. Let $n_{0}$ be the number of zeros of $h_{2}(w)$ in general position in $H(1) / \operatorname{SL}(2, \mathbb{Z})_{2}$ and $n_{2}$ the degree of the zero at $w=(1+i) / 2$. Using Cauchy's theorem for $\left(h_{2}\right)^{-1} d h_{2} / d w$ one obtains

$$
2 N_{4}-n_{0}-n_{2} / 2+N_{2}=0 \text {. }
$$

For $\varepsilon \neq 1$ and for $\varepsilon \equiv 1, D \geqq 12$ one finds $n_{0}=0$, thus $h_{2}(w)$ is given by a product of powers of the functions $\theta_{2}(0, w)^{8}, \theta_{3}(0, w)^{8} \theta_{4}(0, w)^{8}, \theta_{3}(0, w)^{4}+\theta_{4}(0, w)^{4}$, which 
vanish at $i \infty, 0,(1+i) / 2$ respectively and nowhere else. One finds $D=16, \varepsilon \equiv 1$ :

$$
\tilde{H}_{18}^{ \pm}(\zeta, w)=\frac{1}{2} \eta^{-24}(w)\left[ \pm \theta_{1}^{8}(\zeta, w)+\sum_{j=2}^{4} \theta_{j}^{8}(\zeta, w)\right]
$$

$D=12, \varepsilon \equiv 1:$

$$
\begin{aligned}
\tilde{H}_{1 \frac{4}{4}}^{g \pm}(\zeta, w)= & \frac{1}{2} \eta^{-24}(w)\left[ \pm 8 \eta^{6}(w) \theta_{1}^{6}(\zeta, w)+\phi_{2}(w) \theta_{2}^{6}(\zeta, w)\right. \\
& \left.+\phi_{3}(w) \theta_{3}^{6}(\zeta, w)-\phi_{4}(w) \theta_{4}^{6}(\zeta, w)\right],
\end{aligned}
$$

$D=4, \varepsilon$ 丰 1 :

$$
\tilde{H}_{6}(\zeta, w)=\frac{1}{2} \eta^{-12}(w) \sum_{j=2}^{4} \theta_{j}^{2}(\zeta, w) \theta_{j}^{6}(0, w)
$$

where

$$
\left(\phi_{1}, \phi_{2}, \phi_{3}\right)(w)=\left(\theta_{2}^{2}\left(\theta_{3}^{4}+\theta_{4}^{4}\right), \theta_{3}^{2}\left(\theta_{2}^{4}-\theta_{4}^{4}\right), \theta_{4}^{2}\left(\theta_{2}^{4}+\theta_{3}^{4}\right)\right)(0, w) .
$$

For $\varepsilon \equiv 1, D=4,8$ one finds $n_{0} \leqq 1$, i.e. by elementary divisor theory two linearly independent possibilities for $h_{2}(w)$, which yield $D=8$ :

$$
\begin{aligned}
& \tilde{H}_{10}^{ \pm E}(\zeta, w)=\frac{1}{2} \eta^{-24}(w)\left[ \pm 16 \eta^{12}(w) \theta_{1}^{4}(\zeta, w)+E_{4}(w) \sum_{j=2}^{4} \theta_{j}^{4}(\zeta, w) \theta_{j}^{4}(0, w)\right] \\
& \tilde{H}_{10}^{D}(\zeta, w)=\frac{1}{2} \eta^{-24}(w) \sum_{j=2}^{4} \theta_{j}^{4}(\zeta, w) \theta_{j}^{12}(0, w)
\end{aligned}
$$

$D=4$ :

$$
\begin{aligned}
H_{6}^{g \pm}(\zeta, w) & =\frac{1}{2} \eta^{-12}(w)\left[ \pm 8 \eta^{6}(w) \theta_{1}^{2}(\zeta, w)-\phi_{2}(w) \theta_{2}^{2}(\zeta, w)\right. \\
& \left.+\phi_{3}(w) \theta_{3}^{2}(\zeta, w)-\phi_{4}(w) \theta_{4}^{2}(\zeta, w)\right] \\
\tilde{H}_{6}^{g \pm}(\zeta, w)= & \frac{1}{2} \eta^{-24}(w)\left[ \pm 512 \eta^{18}(w) \theta_{1}^{2}(\zeta, w)+\phi_{2}(w)^{3} \theta_{2}^{2}(\zeta, w)\right. \\
& \left.+\phi_{3}(w)^{3} \theta_{3}^{2}(\zeta, w)-\phi_{4}(w)^{3} \theta_{4}^{2}(\zeta, w)\right] .
\end{aligned}
$$

The notation is as follows: The twiddle in $\tilde{H}$ denotes a sign inversion for $d_{F}$, i.e. a partition function constructed from $d_{B}(m, \lambda)+d_{F}(m, \lambda)$ instead of $d_{B}-d_{F}$. The $g$ in $H^{g}$ denotes the presence of ghost states with $\tilde{d}_{B}<0$. In $\tilde{H}_{14}^{g \pm}$ and $\tilde{H}_{6}^{g \pm}$ there are tachyon states. For $H_{6}^{g \pm}$ the spectrum is supersymmetric, but there is a vector ghost at zero mass.

Thus only for $D=8$ one can form a linear combination which satisfies all conditions of the theorem, namely

$$
\left(\tilde{H}_{10}^{D}-\tilde{H}_{10}^{ \pm E}\right) / 16=H_{10}^{ \pm} .
$$

This completes the proof of the theorem.

Equation (*) is very curious, as the left-hand side is closely related to the two possible bosonic sectors of the heterotic string. Let Spin(8) be the usual transverse rotation group and $\operatorname{Spin}(8)^{D}$, $\operatorname{Spin}(8)^{E}$ isomorphic groups which are regularly embedded into the symmetry groups $\operatorname{Spin}^{+}(32), E_{8} \times E_{8}$ respectively of these 
strings. Using the diagonal subgroups of $\operatorname{Spin}(8) \times \operatorname{Spin}(8)^{D}$ and $\operatorname{Spin}(8) \times \operatorname{Spin}(8)^{E}$ as new transverse rotation groups [12] one breaks Lorentz invariance, but obtains the partition functions $\tilde{H}_{10}^{D}, \tilde{H}_{10}^{E}$. Subtraction of these two functions allows the restoration of Lorentz invariance and yields the superstring helicity partition function. In some sense the superstring seems to result from an interference between the $E_{8} \times E_{8}$ and $\operatorname{Spin}^{+}(32)$ compactifications of the string in 26 dimensions. At $m^{2}=0, \tilde{H}_{10}^{ \pm E}$ yields an antisymmetric tensor, $8+1$ vectors, 16 MajoranaWeyl spinors and $\operatorname{dim}\left(E_{8}\right)+\operatorname{dim}(\mathrm{SO}(8))=276$ scalars, whereas $\tilde{H}_{10}^{D}$ yields an antisymmetric tensor, $24+1$ vectors and $\operatorname{dim}(\mathrm{SO}(24))=276$ scalars. Both $\tilde{H}_{10}^{D}$ and $\tilde{H}_{10}^{ \pm E}$ have been constructed in [2], but in an intransparent notation.

To explain the internal excitations arising for $k=1$, note that $\left( \pm \theta_{1}^{r}(\zeta, w)\right.$ $\left.+\theta_{2}^{r}(\zeta, w)\right) / 2,\left(\theta_{3}^{r}(\zeta, w) \pm \theta_{4}^{r}(\zeta, w)\right) / 2$ are the characters of the four irreducible unitary representations of the Kac-Moody algebra $D_{r}^{(1)}$ with central extension $k=1$ [8]. This can be understood in the following way. For the internal contribution to the angular momentum current along the string one may separate left moving and right moving currents. Thus the group $[0, \pi] \rightarrow \operatorname{Spin}(D)$ acting on the string states is lifted to a central extension of the group $(R \bmod 2 \pi) \rightarrow \operatorname{Spin}(D)$ defined on the double cover of $[0, \pi]$. The algebra $D_{r}^{(1)}$ is just the corresponding current algebra.

In general, Jacobi forms of index $k$ are related to representations of $D_{r}^{(1)}$ with central extension $k$. Note, however, that $k=1$ is special:

Proposition 1. Let $H(\zeta, w)$ fulfill conditions of the theorem except possibly $k \leqq 1$. If the string theory is chiral, then $k=1$ and the Lorentz multiplets not invariant under parity are massless Weyl spinors.

Proof. Let parity act on $\zeta$ by $\zeta^{P}=\left(-\zeta_{1}, \zeta_{2}, \zeta_{3}, \ldots\right)$. By Lorentz invariance the difference $A(\zeta, w)-A\left(\zeta^{P}, w\right)$ only gets contributions from massless states. Thus it is independent of $w$ and by modular invariance also of $\zeta$. In particular its index must vanish, which yields $k=1$ for $H(\zeta, w)$. For $A(\zeta, w)-A\left(\zeta^{P}, w\right)=1$ one finds

$$
\sum_{\lambda \in A} d(0, \lambda)\left(\exp (2 \pi i \zeta \lambda)-\exp \left(2 \pi i \zeta^{P} \lambda\right)\right)=Z(\zeta)
$$

Another easy but important result which applies to the superstring is

Proposition 2. Let the modular form $A(w)$ of weight $-D / 2$ be the partition function of a string theory without tachyons. Then either $D=0, A(w)=$ const or $A(w)=0$, i.e. the spectrum is supersymmetric.

Proof. $A(w)$ is a holomorphic modular form. For negative weight such forms must vanish, for weight 0 they must be constant.

The present classification yields much more restrictive results than the one of [2], as we have insisted on the invariance under the full modular group, not just under a subgroup of finite index. This agrees with the known models, though the full Neveu-Schwarz and Ramond models do not have this invariance before their restriction to the superstring.

Unfortunately, our investigation did not yield convincing new candidates for open string models. But in spite of some pathologies the unconventional 
candidates listed in the proofs of Lemmata 6 and 8 may be of interest. Moreover, the relation between the bosonic and superstring sectors of the heterotic string implied by Eq. (*) looks very intriguing.

\section{References}

1. Nahm, W.: Mass spectra of dual strings. Nucl. Phys. B 114, 174 (1976)

2. Nahm, W.: Spin in the spectrum of states of dual models. Nucl. Phys. B 120, 125 (1977)

3. Gross, D., Harvey, J., Martinec, E., Rohm, R.: Heterotic string theory (I). The free heterotic string. Phys. Rev. Lett. 55, 502 (1985); Nucl. Phys. B 256, 253 (1985)

4. Eichler, M., Zagier, D.: On the theory of Jacobi forms. Progress in Mathematics. Boston, MA: Birkhäuser 1985

5. Gunning, R.: Am. J. Math. 78, 357 (1956)

6. Nahm, W.: Functional integrals for the partition functions of dual strings. Nucl. Phys. B 124, 121 (1977)

7. Nahm, W.: A semiclassical calculation of the mass spectrum of relativistic strings. Nucl. Phys. B 81, 164 (1974)

8. Kac, V.: Infinite dimensional Lie algebras. Boston, MA: Birkhäuser 1983

9. Gliozzi, F., Olive, D., Scherk, J.: Supersymmetry, supergravity theories, and the dual spinor model. Nucl. Phys. B 122, 253 (1977)

10. Green, M., Schwarz, J.: Covariant description of superstrings. Phys. Lett. 136B, 367 (1984)

11. Gunning, R.: Lectures on modular forms. Princeton, NJ: Princeton University Press 1962

12. Casher, A., Englert, F., Nicolai, H., Taormina, A.: Consistent superstrings as solutions of the $D=26$ bosonic string theory. Phys. Lett. 162B, 121 (1985)

Communicated by A. Jaffe

Received January 3, 1986 
